\title{
Generation of Naïve Blastoderm Explants from Zebrafish Embryos
}

\author{
Alyssa Alaniz Emig ${ }^{1}$, Margot L. K. Williams ${ }^{1}$ \\ ${ }^{1}$ Center for Precision Environmental Health and Department of Molecular and Cellular Biology, Baylor College of Medicine
}

\section{Corresponding Author}

Margot L. K. Williams

Margot.Williams@BCM.edu

\section{Citation}

Alaniz Emig, A.,

Williams, M.L.K. Generation of Naïve Blastoderm Explants from Zebrafish Embryos. J. Vis. Exp. (173), e62797, doi:10.3791/62797 (2021).

\section{Date Published}

July 30,2021

DOI

$10.3791 / 62797$

URL

jove.com/video/62797

\section{Abstract}

Due to their optical clarity and rapid development, zebrafish embryos are an excellent system for examining cell behaviors and developmental processes. However, because of the complexity and redundancy of embryonic signals, it can be challenging to discern the complete role of any single signal during early embryogenesis. By explanting the animal region of the zebrafish blastoderm, relatively naïve clusters of embryonic cells are generated that can be easily cultured and manipulated ex vivo. By introducing a gene of interest by RNA injection before explantation, one can assess the effect of this molecule on gene expression, cell behaviors, and other developmental processes in relative isolation. Furthermore, cells from embryos of different genotypes or conditions can be combined in a single chimeric explant to examine cell/tissue interactions and tissue-specific gene functions. This article provides instructions for generating zebrafish blastoderm explants and demonstrates that a single signaling molecule - a Nodal ligand - is sufficient to induce germ layer formation and extension morphogenesis in otherwise naïve embryonic tissues. Due to their ability to recapitulate embryonic cell behaviors, morphogen gradients, and gene expression patterns in a simplified ex vivo system, these explants are anticipated to be of great utility to many zebrafish researchers.

\section{Introduction}

A perennial goal of the developmental biology field is to unravel the complexity of developing embryos to understand the origin of animal form and function. Even early embryos contain a complex medley of signaling molecules, cell and tissue interactions, and mechanical forces, all subject to strict spatial and temporal regulation. For this reason, it is often challenging to pinpoint the precise role of a particular signal in a developmental process of interest. By removing embryonic tissues from their endogenous environment, embryo explantation creates a simplified platform in which to discern the developmental roles of individual tissues and molecules in relative isolation. Explantation techniques are perhaps best known in Xenopus laevis, where they have been used to study tissue induction, cell signaling, cell adhesion, 
and morphogenesis, among other processes ${ }^{1,2,3,4}$. Socalled animal cap explants, in which the animal region of blastula stage Xenopus embryos is isolated before inductive interactions ${ }^{5,6,7}$, are a widespread and powerful explant technique. Unmanipulated animal caps are fated to become ectoderm ${ }^{7,8}$. Still, they are competent to respond to several inductive factors, allowing them to form tissues of all three germ layers and undergo tissue-appropriate morphogenetic movements $9,10,11$. However, limited genetic tools and suboptimal suitability for live imaging prevent the use of Xenopus animal cap explants for many developmental biologists. By explanting blastoderm cells from zebrafish embryos, researchers can combine the utility of the animal cap assay with the optical clarity, abundance of genetic tools, and other experimental advantages of the zebrafish model system.

To date, researchers have made use of two flavors of zebrafish explants: so-called pescoids and the blastoderm explants. In the pescoid model, the entire blastoderm, including the marginal zone, is isolated from the yolk and allowed to develop ex vivo without the extraembryonic yolk syncytial layer $(\mathrm{YSL})^{12,13}$. In this way, pescoids bear a notable resemblance to the Fundulus explants generated decades ago by Jane Oppenheimer and J.P. Trinkaus ${ }^{14,15}$. These explants recapitulate many aspects of embryonic patterning and morphogenesis ${ }^{12,13}$. However, because these isolates contain endogenous signaling centers (the embryonic margin), they are not simplified with regard to their molecular milieu. Alternatively, researchers can generate relatively naïve zebrafish blastoderm explants by excluding the marginal zone $^{16,17,18,19,20,21}$. Unmanipulated zebrafish blastoderm explants express high levels of bone morphogenetic protein (BMP) morphogens ${ }^{19}$ and give rise to non-neural ectoderm and enveloping layer (EVL) when cultured ex vivo ${ }^{18}$.
However, they recapitulate many aspects of axial patterning and morphogenesis in response to exogenous signaling gradients $19,20,21$, similar to Xenopus animal caps. For this reason, blastoderm explants are an advantageous model to study the role of a given morphogen (or morphogens) in germ layer specification, morphogenetic cell movements, and signaling gradients within a simplified signaling environment. Furthermore, blastoderms from embryos of different genotypes or conditions can be combined in a single chimeric explant ${ }^{19,21}$ to investigate cell/tissue autonomy and inductive interactions.

Zebrafish blastoderm explants can be used to investigate the role of embryonic signals (for example, Nodal) in morphogenesis and tissue specification during gastrulation. By injecting synthetic ndr2 RNA (encoding a Nodal ligand) at the single-cell stage, Nodal signaling is activated throughout the blastoderm of the embryo. Explants from these embryos generate Nodal signaling gradients, form all three germ layers, and undergo convergence and extension (C\&E) gastrulation movements as seen in intact embryos ${ }^{20}$. Additionally, chimeric explants are used to illustrate the ability of mesoderm tissues to induce neuroectoderm from uninjected (naïve) blastoderm. This protocol provides instructions for creating zebrafish blastoderm explants and demonstrates their utility in defining the role of Nodal signaling in tissue induction and morphogenesis.

\section{Protocol}

\section{Prepare the reagents and supplies}

1. Reagent preparation

1. Prepare $500 \mathrm{~mL}$ of $3 x$ Danieau's solution (Solution 1, Table 1). 
2. Prepare $1 \mathrm{~L}$ of egg water (Solution 2, Table 1).

3. Prepare a $1.2 \%$ solution of agarose in egg water. Melt the agarose entirely in the microwave, and then cool to $55^{\circ} \mathrm{C}$ in a water bath.

4. Prepare $4 \mathrm{~mL}$ explant media (Solution 3, Table 1, modified slightly from ${ }^{19,21}$ ) per experimental condition.

NOTE: Remember to account for at least one well of explants from uninjected (or control injected) embryos when calculating the required volume.

1. Sanitize the workspace with $70 \%$ ethanol.

2. Remove cell culture media from $4{ }^{\circ} \mathrm{C}$ and spray/ wipe with $70 \%$ ethanol.

3. Make explant media and place in the 28.5 ${ }^{\circ} \mathrm{C}$ incubator to warm while the embryos are injected.

NOTE: Always include age-matched, intact sibling embryos for staging purposes. Dechorionate these embryos and culture them on agarose-coated plates in $0.3 x$ Danieau's solution (Solution 4, Table 1).

5. Remove pronase aliquots $(1 \mathrm{~mL}$ at $20 \mathrm{mg} / \mathrm{mL}$ ) from $-20{ }^{\circ} \mathrm{C}$ and allow to thaw on ice. Thaw one $1 \mathrm{~mL}$ aliquot for every three experimental conditions.

2. Prepare agarose plates

1. Make the injection plates.

1. Fill a $100 \mathrm{~mm} \times 15 \mathrm{~mm}$ plastic Petri dish halfway with molten agarose in egg water.

2. Gently place the injection mold on top of the molten agarose at a $45^{\circ}$ angle and lower it gradually into the agarose, ensuring that no bubbles are trapped underneath. Let it cool completely.

3. Remove the mold. Use the plate immediately or save for later by adding $2 \mathrm{~mL}$ of egg water, wrapping the plate, and storing it at $4{ }^{\circ} \mathrm{C}$. Warm the plate for $15-30 \mathrm{~min}$ in the $28.5^{\circ} \mathrm{C}$ incubator before injection.

2. Make explant cutting plates.

1. Add $3 \mathrm{~mL}$ of molten $1.2 \%$ agarose in egg water to a $60 \mathrm{~mm} \times 15 \mathrm{~mm}$ Petri dish, ensuring that the entire bottom of the well is coated. Let it cool completely.

3. Coat culture plates with agarose.

1. For each experimental condition, dispense 1 $\mathrm{mL}$ of molten $1.2 \%$ agarose in egg water into one well of a 6-well plate, ensuring that the entire bottom of the well is coated. Let it cool completely.

4. For making chimeric explants, create an explant cutting dish with small wells by adding twelve $1 \mathrm{~mm}$ glass beads to molten agarose in a $60 \mathrm{~mm} \times 15 \mathrm{~mm}$ Petri dish. Remove the beads with forceps once the agarose cools completely.

\section{Inject embryos with RNA}

1. Wearing gloves, remove an aliquot of synthetic $n d r 2$ mRNA from storage at $-80{ }^{\circ} \mathrm{C}$ and immediately place it on ice.

2. Prepare the injection needle.

1. Fill a pulled glass capillary needle with RNA. Place the filled needle into a micro-manipulator and break the tip of the needle with forceps. 
2. Calibrate injection volume using a stage micrometer with a drop of mineral oil, adjusting injection time and pressure on the pneumatic injector to achieve a bolus of the desired size. A bolus with a diameter of $120 \mu \mathrm{m}$ has a volume of $1 \mathrm{~nL}$.

NOTE: The desired volume of the bolus will depend on the concentration of the RNA and the desired dose per embryo. For example, if RNA is aliquoted at $10 \mathrm{ng} / \mu \mathrm{L}$, inject $1 \mathrm{~nL}$ to achieve a final amount of $10 \mathrm{pg}$.

1. Keep the RNA needle tip submerged in the oil until ready to inject.

3. Load the embryos and inject.

1. Pull the dividers in breeding tanks, allow fish to spawn for 10-15 min, and collect the embryos using a tea strainer.

2. Load the embryos into the injection plate using a Pasteur pipette and pipette pump, and then use a gloved finger to press the eggs into the troughs gently.

3. Inject $10 \mathrm{pg} n d r 2$ RNA into the yolk of single-cell embryos until the desired number of embryos is reached or until embryos begin to divide.

NOTE: Do not inject after the single-cell stage to ensure even distribution of the RNA throughout the embryo.

4. Wash the embryos out of the injection plate into a labeled $100 \mathrm{~mm} \times 15 \mathrm{~mm}$ Petri dish with a gentle stream of egg water from a squeeze bottle.

NOTE: Always keep a group of age-matched, uninjected siblings as controls.
5. Place the embryos into the $28.5{ }^{\circ} \mathrm{C}$ incubator until they reach the 128-cell stage. Remove unfertilized eggs and dead embryos from the dish.

\section{Dechorionate the embryos}

1. Once the embryos have reached the 128-cell stage, place them into labeled glass Petri dishes and decant as much egg water as possible from them.

2. Label glass crystalizing dishes with lab tape (corresponding to small dish names) and fill $2 / 3$ of the way with egg water. Place these dishes next to the dissecting microscope for quick accessibility.

3. Add $1 \mathrm{~mL}$ of pronase stock ( $20 \mathrm{mg} / \mathrm{mL}$, thawed on ice) to $15 \mathrm{~mL}$ of $3 x$ Danieau's solution in a $50 \mathrm{~mL}$ conical tube. NOTE: This amount is sufficient for up to three experimental conditions. Increase the volume of pronase and 3x Danieau's solution for additional explant conditions.

CAUTION: Pronase is an irritant; hence wear gloves when handling.

4. Add at least $5 \mathrm{~mL}$ of pronase solution to each glass Petri dish containing embryos.

5. Agitate the glass dishes in a circular motion, monitoring the progress of dechorionation consistently under a dissecting microscope.

6. Once the chorions begin to wrinkle and 1-2 embryos are out of their chorions, carefully dunk the glass Petri dish containing pronase and the embryos into the corresponding glass crystallizing dish containing egg water.

7. Wash the dechorionated embryos. 
1. Wash the embryos three times with egg water by gently adding and then decanting egg water from the dish.

2. The third and final wash is with $0.3 x$ Danieau's solution.

NOTE: If the embryos still have chorions after washing, gently pipette the embryos until the chorions are removed or let them sit in the wash (egg water or $0.3 x$ Danieau's solution) for a minute or two and gently agitate with circular motions.

8. Cover dechorionated embryos with a Petri dish lid and return them to the incubator $\left(28.5^{\circ} \mathrm{C}\right)$ until they reach the 256-cell stage.

\section{Cut explants}

1. Fill the agarose-coated $60 \mathrm{~mm} \times 15 \mathrm{~mm}$ Petri dish with 3x Danieau's solution.

2. Once the embryos are at the 256-cell stage, transfer them into the agarose-coated plate containing $3 x$ Danieau's solution, lining them up along the center of the dish.

3. Cut the explants using forceps (Figure 1).

1. Use one pair of forceps, held closed, to stabilize the embryo and use the other to cut through the blastoderm at approximately half of its height (from margin to animal pole) (Figure 1A).

2. To cut, gently squeeze the blastoderm cells with one pair of forceps. Then, take the stabilizing forceps and run them along the other forceps to slice approximately halfway across the blastoderm (Figure 1B).
3. Rotate the embryo, placing the forceps into the existing cut, and then sever the remaining blastoderm orthogonal to the first cut (Figure 1C).

4. Keep explants in $3 x$ Danieau's solution for at least 5 min to heal, then transfer them to the well of a 6-well plate coated with agarose and filled with $4 \mathrm{~mL}$ of explant media. NOTE: Cut explants from uninjected (or control injected) siblings as negative controls. If explants are performed correctly, these explants will neither extend nor express markers of endoderm, mesoderm, or neuroectoderm.

5. Place the explant culture plates into the $28.5^{\circ} \mathrm{C}$ incubator until the desired timepoint/stage (determined from intact siblings) is reached.

NOTE: If treating explants with a compound, such as a small molecule inhibitor, the desired concentration can be added directly to the explant media within the wells at desired time points. Remember to include the volume of agarose when calculating concentrations. (Example: 1 $\mathrm{mL}$ agarose $+4 \mathrm{~mL}$ explant media $=5 \mathrm{~mL}$ total volume per well).

\section{Prepare chimeric explants}

1. In place of a regular agarose coated plate, cut chimeric explants in a dish with agarose molded into twelve small, shallow wells using $1 \mathrm{~mm}$ glass beads (section 1.2.4). Fill this plate with $3 x$ Danieau's solution.

NOTE: Chimeric explants are generated from blastoderm cells of two embryos of different genotypes or conditions. Ensure that these conditions can be distinguished from one another by the expression of transgenic or injected fluorescent markers.

1. Prepare by adding twelve embryos of one genotype/ condition to the left side of the plate and twelve 
embryos of the other genotype/condition to the right side of the plate.

2. Move one embryo of each condition into the center of the plate, near one of the 12 wells.

3. Using forceps, cut an explant from each embryo as described for single embryo explants (step 4.3).

4. Quickly press the cut edges of the two explants together within the shallow well using forceps to allow the two halves to heal together into a single explant.

5. Continue with the remaining eleven wells within the plate. Once explants are healed, transfer them to the well of a 6-well plate coated with agarose and filled with $4 \mathrm{~mL}$ of explant media. Repeat until the desired number of explants is achieved.

\section{Culture and/or image the fixed explants}

1. Culture explants in the $28.5{ }^{\circ} \mathrm{C}$ incubator until intact sibling embryos reach the desired stage.

2. Live explants can be mounted for continuous timelapse imaging, imaged periodically throughout the culture period, or imaged live at the experimental endpoint.

3. Fix the explants if desired. Once the explants reach the desired endpoint, note the stage of intact embryo siblings and place the explants into a glass scintillation vial with $1 \mathrm{~mL}$ of $4 \%$ paraformaldehyde in PBS. Fix overnight on a shaker at $4{ }^{\circ} \mathrm{C}$.

CAUTION: Paraformaldehyde is toxic. Wear gloves when handling this chemical and dispose of it via methods approved by each institution.

1. After fixation, rinse explants six times, $15 \mathrm{~min}$ each with PBS $+0.1 \%$ Tween-20, and dehydrate gradually into methanol. Store explants at -20
${ }^{\circ} \mathrm{C}$ for later analysis by the whole-mount in situ hybridization, immunofluorescent staining, etc.

\section{Representative Results}

Nodal ligands drive germ layer formation and C\&E of zebrafish blastoderm explants

Control explants cut from uninjected wild-type (WT) embryos or those injected with $50 \mathrm{pg}$ of mRNA encoding green fluorescent protein (GFP) remained rounded throughout the culture period (Figure 2A-C) and failed to express markers of mesoderm, endoderm, or neuroectoderm (Figure 3C) ${ }^{20}$. Together, these indicate an absence of the morphogenesis and germ layer formation that characterize vertebrate gastrulation. However, explants cut from embryos injected with $10 \mathrm{pg}$ of $n d r 2$ mRNA became highly elongated after 8-9 $\mathrm{h}$ in culture (Figure 2D). Live time-lapse imaging of these explants by differential interfering contrast (DIC) microscopy revealed that extension onsets at or around 8 h post-fertilization (hpf) (Figure $2 F$ ), the same time that C\&E morphogenesis begins in intact zebrafish embryos ${ }^{22}$. Explants cut from MZoep-/- embryos, which lack the essential tdgf1 Nodal co-receptor ${ }^{23}$, failed to extend in response to $n d r 2$ injection (Figure 2E), demonstrating that Nodal activity is critical for this ex vivo morphogenesis. In addition, whole-mount in situ hybridization further showed that $n d r 2-$ expressing explants express markers of neuroectoderm (sox2) and several mesoderm sub-types (tbxta, noto, tbx16) (Figure 2G), as well as endoderm and the embryonic organizer $^{20}$.

Nodal signaling is not required for neuroectoderm induction by mesoderm

Nodal signaling activity is essential for induction of endoderm and most mesoderm but is dispensable for neuroectoderm specification within zebrafish gastrulae $e^{23,24}$. 
While uninjected zebrafish blastoderm explants did not differentiate into neuroectoderm (Figure $3 \mathrm{C}^{18}$ ), explants from embryos injected with $10 \mathrm{pg}$ ndr2 exhibited robust expression of the neuroectoderm marker sox 2 in distinct stripes along the long axis of the explant (Figure 2G), indicating that Nodal activity is required for neuroectoderm formation ex vivo. It has long been known that mesodermal tissues can induce neural tissue $25,26,27,28,29$, including in zebrafish blastoderm explants ${ }^{17}$. However, it is unclear whether neuroectoderm formation in this explant system requires Nodal signaling directly or whether exogenous Nodal ligands induce mesoderm that then induces neural tissues secondarily.

Chimeric explants containing prospective mesoderm and neuroectoderm portions from two different embryos were generated to test whether Nodal signaling is required tissueautonomously for neuroectoderm specification ex vivo. The mesoderm portion of each explant was cut from an otherwise WT embryo expressing a mesoderm-specific transgenic GFP reporter, $\operatorname{Tg}[\operatorname{Ihx} 1 \mathrm{a}: \mathrm{eGFP}]^{30}$, injected with a high dose (100 pg) of ndr2 (Figure 3A). The putative neuroectoderm portion of each explant was cut from either a control WT embryo or Nodal signaling deficient MZoep-/- embryo injected only with mRNA encoding the fluorescent nuclear marker H2BRFP (Figure 3A). Each chimeric explant was generated by combining one blastoderm from each of these two conditions, which were assayed for the expression of tissue-specific markers by whole-mount in situ hybridization at $12 \mathrm{hpf}$.
The majority of single-embryo explants from embryos injected with $100 \mathrm{pg}$ ndr2 expressed little or no sox2, and expressed markers of mesoderm, including tbxta and the Ihx1a:gfp reporter-throughout the explant (Figure 3B,G). Uninjected WT blastoderm (of the type that comprises the prospective neuroectoderm portion of chimeric explants) expressed neither mesoderm markers nor sox2 when cultured as a single explant, indicating a lack of neuroectoderm and mesoderm specification (Figure $\mathbf{3 C , H}$ ). Single-embryo explants from MZoep-/- embryos similarly lacked expression of both neuroectoderm and mesoderm markers, even when injected with ndr2 (Figure 3D). However, when uninjected WT blastoderms were combined with mesoderm induced by high doses of Nodal ligands, these chimeric explants expressed both mesoderm markers and sox 2 robustly (Figure 3E,I). These results demonstrate that, as previously observed $^{17,26,27,29}$, mesoderm can induce neural fate in cells that would otherwise become non-neural ectoderm. To test whether Nodal signaling is required directly within the prospective neuroectoderm portion of these explants for their neural induction, chimeric explants were created from WT blastoderms injected with $100 \mathrm{pg} n d r 2$ and blastoderms from MZoep-/- embryos (Figure $3 \mathbf{J})$. Despite their inability to receive Nodal signals from the neighboring mesodermal portion, these explants expressed sox 2 to a similar degree as WT control chimeras (Figure 3F). This result demonstrates that consistent with intact embryos in which neural tissues are specified in the absence of Nodal activity, Nodal signaling is not required tissue-autonomously for neuroectoderm induction ex vivo. 


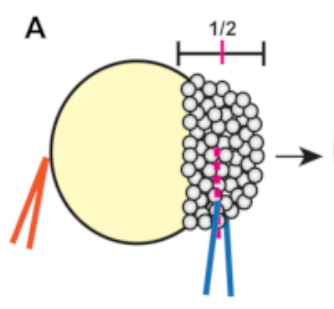

B

C

D

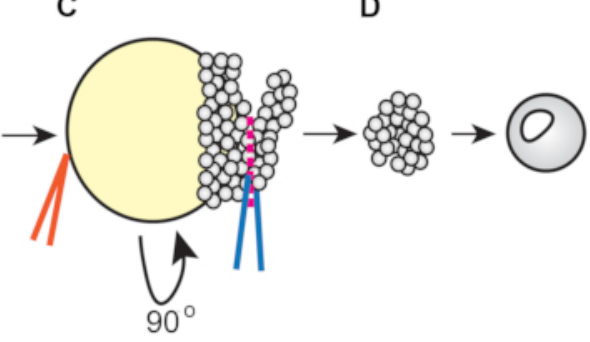

Figure 1: Procedure for zebrafish blastoderm explantation (step 4.3). (A) Hold the forceps in the non-dominant hand (orange) closed against the yolk to stabilize the embryo while pinching the blastoderm at approximately $1 / 2$ of its height using the forceps in the dominant hand (blue). (B) Run the orange forceps along the edge of the blue forceps gripping the embryo to slice through the blastoderm so that the first cut reaches approximately halfway across the blastoderm. (C) Rotate the embryo $90^{\circ}$, then place the blue forceps inside (but orthogonal to) the original cut and pinch to sever the remaining blastoderm. (D) Allow explanted blastoderm cells to heal in 3x Danieau's solution for approximately 5 min before transferring into explant media. Please click here to view a larger version of this figure. 


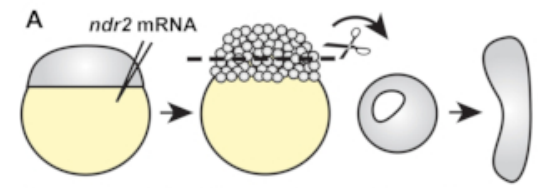

B Uninjected WT

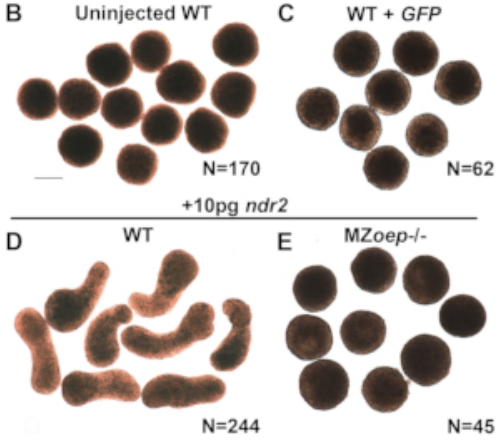

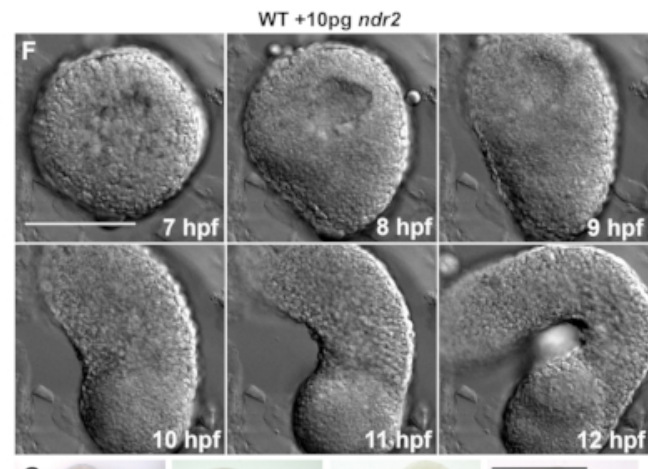

G

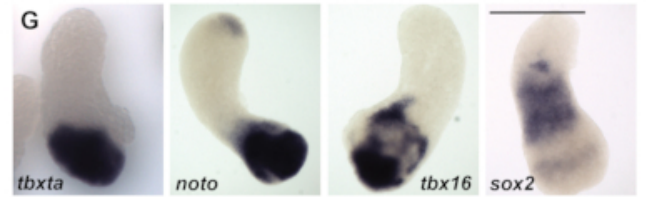

Figure 2 (modified from ${ }^{20}$ ): Nodal ligands promote C\&E morphogenesis and germ layer formation in zebrafish blastoderm explants. (A) Diagram of injection and explantation of zebrafish embryos. (B-E) Representative bright-field images of live blastoderm explants of the indicated conditions/genotypes at the of equivalent the 2-4 somite stage. $\mathrm{N}=$ number of explants from two to four independent trials. (F) Time-lapse DIC series of a representative explant from a WT embryo injected with $10 \mathrm{pg} n d r 2$ RNA. (G)Representative images of the whole-mount in situ hybridization for the transcripts indicated in explants from WT embryos injected with $10 \mathrm{pg} n d r 2$ RNA. Scale bars are $200 \mu \mathrm{m}$. Please click here to view a larger version of this figure. 


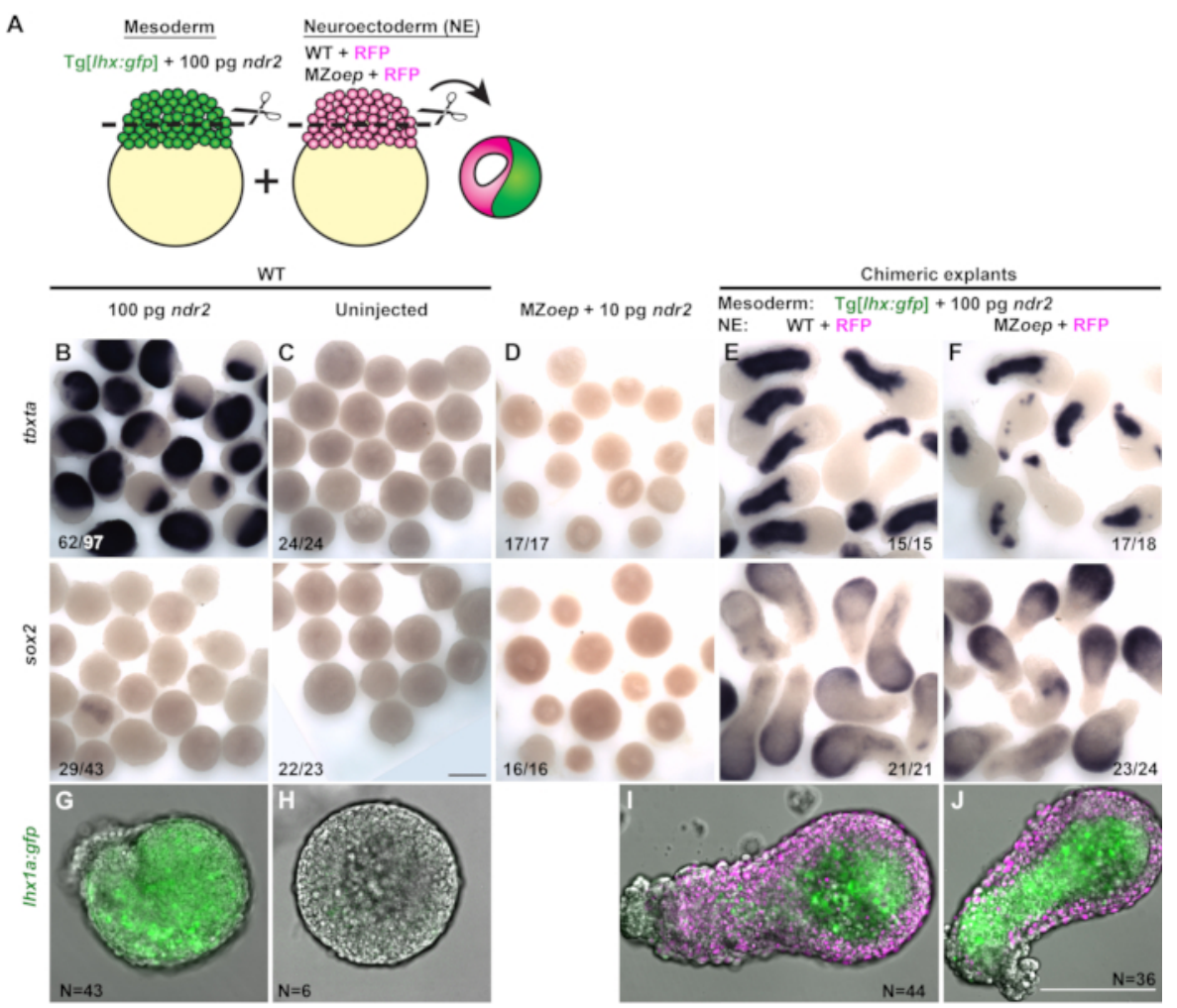

Figure 3 (Modified from ${ }^{31}$ ): Chimeric explants reveal that neuroectoderm specification does not require tissueautonomous Nodal signaling ex vivo. (A) Diagram of the procedure to generate chimeric zebrafish explants. (B-F) Wholemount in situ hybridization for the mesoderm marker tbxta (top) and neuroectoderm marker sox2 (bottom) in explants from WT embryos injected with 100 pg ndr2 RNA (B), uninjected WT controls (C), MZoep-/- injected with 10 pg ndr2 (D), and chimeric explants containing neuroectoderm portions from WT (E) or MZoep-/- (F) embryos at the equivalent of the 2-4 somite stage. Fractions indicate the number of explants with the phenotype shown over the total number of explants examined. (G-J) Representative images of live Tg[lhx1a:gfp] explants from a single embryo (G-H) or combined with H2Bexpressing blastoderms (I-J, magenta) of the conditions indicated at the equivalent of the 2-4 somite stage. $\mathrm{N}=$ number of explants from three independent trials. Scale bars are $200 \mu \mathrm{m}$. Please click here to view a larger version of this figure. 


\begin{tabular}{|c|c|c|c|c|}
\hline & Solution 1 & Solution 2 & Solution 3 & Solution 4 \\
\hline Solution & 3x Danieau's & Egg Water & Explant Media & 0.3x Danieau's \\
\hline \multirow[t]{9}{*}{ Ingredients } & $174 \mathrm{mM} \mathrm{NaCl}$ & $60 \mu \mathrm{g} / \mathrm{mL}$ sea salts & $\begin{array}{c}\text { DMEM/F12 + } 2.5 \\
\text { mM L-Glutamine } \\
\text { and } 15 \text { mM HEPES }\end{array}$ & $17.4 \mathrm{mM} \mathrm{NaCl}$ \\
\hline & $2.1 \mathrm{mM} \mathrm{KCl}$ & $1 \mathrm{~L}$ distilled water & $\begin{array}{l}3 \% \text { total volume of } \\
\text { Newborn Calf Serum } \\
\text { or Fetal Bovine Serum }\end{array}$ & $0.21 \mathrm{mM} \mathrm{KCl}$ \\
\hline & $1.2 \mathrm{mM} \mathrm{MgSO}_{4} .7 \mathrm{H}_{2} \mathrm{O}$ & & $\begin{array}{c}\text { 1:200 Penicillin (50 } \\
\text { units } / \mathrm{mL}) \text {-Streptomycin } \\
(50 \mu \mathrm{g} / \mathrm{mL})\end{array}$ & $0.12 \mathrm{mM} \mathrm{MgSO} 4 \cdot 7 \mathrm{H}_{2} \mathrm{O}$ \\
\hline & $\begin{array}{c}1.8 \mathrm{mM} \\
\mathrm{Ca}\left(\mathrm{NO}_{3}\right)_{2} \cdot 4 \mathrm{H}_{2} \mathrm{O}\end{array}$ & & & $\begin{array}{c}0.18 \mathrm{mM} \\
\mathrm{Ca}\left(\mathrm{NO}_{3}\right)_{2} \cdot 4 \mathrm{H}_{2} \mathrm{O}\end{array}$ \\
\hline & $15 \mathrm{mM}$ HEPES & & Example: & $1.5 \mathrm{mM}$ HEPES \\
\hline & Distilled water & & $\begin{array}{l}4 \mathrm{~mL} / \text { condition } \times 9 \\
\text { Conditions }=36 \mathrm{~mL}\end{array}$ & Distilled water \\
\hline & & & $\begin{array}{c}1.08 \mathrm{~mL} \text { Newborn Calf } \\
\text { Serum (NCS, aliquoted } \\
\text { in }-20^{\circ} \mathrm{C}(3 \%)\end{array}$ & \\
\hline & & & $\begin{array}{l}0.18 \mathrm{~mL} 200 x \text { Pen- } \\
\text { Strep (PS, aliquoted } \\
\left.\text { in }-20^{\circ} \mathrm{C}\right)(1: 200)\end{array}$ & \\
\hline & & & 35 mL DMEM/F12 & \\
\hline
\end{tabular}

Table 1.

\section{Discussion}

This article has described how to generate zebrafish blastoderm explants and discussed two practical applications of these explants in addressing the role of Nodal morphogen signaling in gastrulation. This method of cutting and culturing explants provides a blank slate of naïve cells that can be

manipulated using RNA injections and treatment with small molecule compounds to investigate a molecular pathway of interest.

\section{Critical steps}


There are four steps in this protocol that are particularly critical for its success. The first is injecting the embryos with the appropriate amount of Nodal. This protocol recommends 10 pg of $n d r 2$ RNA, and although a range of doses promotes extension, too much or too little Nodal will prevent optimal explant extension ${ }^{20}$. The second step is dechorionating the embryos. If the embryos remain in pronase for too long, the yolks will burst, and the embryos will not be viable to cut. If they are not in the pronase long enough, the chorions will not be loosened by washing and will instead require time-consuming manual dechorionation. The third critical step is cutting the explants. Cutting in $3 x$ Danieau's solution is recommended, as the lower salt content of $0.3 x$ Danieau's solution or egg water does not promote healing and survival of explants.

Additionally, the explants must be cut at approximately half the height of the blastoderm to ensure the naivety of the cells. If they are cut too close to the yolk, they will contain signals from the margin (including endogenous Nodal) that promote tissue specification and morphogenesis. The fourth and final critical step is in the healing of chimeric explants. Two explants will not fuse to form chimeras unless their cut edges are gently pressed together immediately after they are cut.

\section{Modifications and troubleshooting}

The critical steps described above provide opportunities for troubleshooting. Some common issues and proposed solutions are presented below.

If explants are not extending in the presence of Nodal signaling, there are some possible solutions. (A) Inject embryos at the single-cell stage to ensure that RNA is evenly dispersed throughout the entire embryo. (B) Avoid injecting too much nodal RNA by ensuring that the injected volume is correct using a micrometer to measure the injection bolus. (C) Avoid injecting too little nodal RNA by measuring its concentration to ensure it has not degraded. (D) Keep some age-matched intact siblings to infer the equivalent stage of the explants. Explants achieve maximum extension when intact siblings reach the $2-5$ somite stage. If the explants are collected too early, then the optimal extension will not be reached.

If the yolks are bursting after dechorionation, and the embryos are not viable to cut, remove the embryos from pronase solution once the chorions begin to crinkle and 1-2 embryos shed their chorion. Then, rinse immediately in egg water.

If the explants appear bubbly around the edges, there are some solutions. (A) Cut explants only within a specific timeframe of development. Although explants cut at any stage from 128- to 1000-cell stages can survive and extend in culture, those cut at 256 - to 512 -cell stages tend to be the most robust. (B) Ensure that explants are cut in 3x Danieau's solution to ensure proper healing. (C) Cut explants cleanly but gently. Avoid stretching or pulling the cells apart during the cutting process.

If the uninjected control explants are extending, explants were likely to cut too close to the yolk. For explants to be naïve, ensure that the cuts are made halfway between the yolk and the top of the blastoderm.

If the chimeric explants fail to fuse, it is likely because the tendency of explants in 3x Danieau's solution once cut is to round up and heal over the cut edge. To ensure that the two blastoderms heal to each other rather than themselves, press them together immediately after cutting. Use forceps to apply gentle pressure to the newly joined blastoderms within the agarose well to encourage them to heal together. 


\section{Limitations}

While these explants are a valuable tool to study the role of a given morphogen (or another molecule of interest) in relative isolation, observations made in any ex vivo model must be interpreted with care. Explants exhibit C\&E morphogenesis that is very similar to that observed in vivo ${ }^{20}$, but they do not recapitulate all aspects of gastrulation, for example, epiboly movements. They also lack many other regulatory factors and signaling molecules that are present within an intact embryo. While this is a significant experimental advantage of explants, it can also lead to conclusions that do not hold in vivo. For example, since explants that do not receive exogenous Nodal ligands fail to express neuroectoderm markers, one might conclude from explants alone that Nodal signaling is required for neuroectoderm specification. However, neuroectoderm is formed within intact embryos lacking all Nodal signaling 23,24 , demonstrating the vital role of other signaling molecules in neural specification ${ }^{32}$. Explants can tell us what a morphogen is capable of in an isolated environment. Still, all such findings should be confirmed in/compared with intact embryos for results to be interpreted thoroughly. In other words, explants cannot take the place of a developing embryo. Instead, they are a supplementary tool to identify the role and relationship of a morphogen with the surroundings. With these limitations in mind, zebrafish blastoderm explants are a valuable tool for many research questions.

\section{Significance with respect to existing methods}

With renewed interest in synthetic embryology, several ex vivo and in vitro approaches are regularly employed to model aspects of embryonic development. For example, 2- and 3-dimensional gastruloids composed of mouse or human embryonic / induced pluripotent stem cells can be coaxed, through the application of exogenous signaling molecules, to recapitulate some of the patterning and/ or morphogenetic events of gastrulation, segmentation, and neurulation $33,34,35,36,37$. Although powerful, these methods require laborious and prolonged culture methods to both continuously maintain pluripotent stem cells and to grow gastruloids, which take many days to reach gastrulation stages. By contrast, zebrafish explants require no maintenance of stem cell cultures, as embryos are simply collected as needed. They are relatively simple to generate and reach gastrulation stages within hours, the same as zebrafish embryos. This highlights another advantage of zebrafish explants, their intact developmental clock. Because the developmental age of embryonic and induced pluripotent stem cells can be variable and highly debated, embryonic explants are perhaps better suited to investigate temporal regulation of development. Finally, while pescoid zebrafish explants (which contain the embryonic margin) similarly extend in culture ${ }^{12,13}$, they do so in response to endogenous signaling centers. Instead, the explants described here enable researchers to investigate molecules of interest with relatively little interference from such embryonic signals.

\section{Potential future applications}

Here, explants were used to demonstrate that Nodal signaling is necessary and sufficient for C\&E morphogenesis. Still, it is anticipated that they can and will be used to discern the role of many different molecules in many other developmental processes, for example, regulation of gene expression, signaling gradients, and additional morphogenetic programs. Additionally, because these explants are viable until at least $24 \mathrm{hpf}^{19}$, it can be expected that their utility will extend beyond gastrulation into processes such as segmentation and organogenesis, any process in which researchers desire a developmental blank slate. 


\section{Disclosures}

The authors declare that they have no competing financial interests.

\section{Acknowledgments}

This work was supported by NICHD R00HD091386 to MLKW and by NIEHS T32ES027801 to AAE.

\section{References}

1. Green, J. B., New, H. V., Smith, J. C. Responses of embryonic Xenopus cells to activin and FGF are separated by multiple dose thresholds and correspond to distinct axes of the mesoderm. Cell. 71 (5), 731-739 (1992).

2. Sudarwati, S., Nieuwkoop, P. D. Mesoderm formation in the anuranXenopus laevis (Daudin). Wilhelm Roux Archiv fur Entwicklungsmechanik der Organismen. 166 (3), 189-204 (1971).

3. Gurdon, J. B., Fairman, S., Mohun, T. J., Brennan, S. Activation of muscle-specific actin genes in Xenopus development by an induction between animal and vegetal cells of a blastula. Cell. 41 (3), 913-922 (1985).

4. Keller, R., Danilchik, M. Regional expression, pattern and timing of convergence and extension during gastrulation of Xenopus laevis. Development. 103 (1), 193-209 (1988).

5. Ariizumi, T. et al. Isolation and differentiation of Xenopus animal cap cells. Current Protocols in Stem Cell Biology. Chapter 1 Unit 1D.5 (2009).

6. Asashima, M., Grunz, H. Effects of inducers on inner and outer gastrula ectoderm layers of Xenopus laevis. Differentiation. 23 (3), 206-212 (1983).
7. Jones, E. A., Woodland, H. R. Development of the ectoderm in Xenopus: tissue specification and the role of cell association and division. Cell. 44 (2), 345-355 (1986).

8. Keller, R. E. Vital dye mapping of the gastrula and neurula of Xenopus laevis. I. Prospective areas and morphogenetic movements of the superficial layer. Developmental Biology. 42 (2), 222-241 (1975).

9. Sokol, S., Wong, G. G., Melton, D. A. A mouse macrophage factor induces head structures and organizes a body axis in Xenopus. Science. 249 (4968), 561-564 (1990).

10. Thomsen, G. et al. Activins are expressed early in Xenopus embryogenesis and can induce axial mesoderm and anterior structures. Cell. 63 (3), 485-.493 (1990).

11. Howard, J. E., Smith, J. C. Analysis of gastrulation: different types of gastrulation movement are induced by different mesoderm-inducing factors in Xenopus laevis. Mechanisms of Development. 43 (1), 37-48 (1993).

12. Fulton, T. et al. Axis specification in Zebrafish is robust to cell mixing and reveals a regulation of pattern formation by morphogenesis. Current Biology. 30 (15), 3063-3064 (2020).

13. Schauer, A., Pinheiro, D., Hauschild, R., Heisenberg, C.-P. Zebrafish embryonic explants undergo genetically encoded self-assembly. eLife. e55190 (2020).

14. Oppenheimer, J. M. The development of isolated blastoderms of Fundulus heteroclitus. The Journal of Experimental Zoology. 72 (2), 247-269 (1936).

15. Trinkaus, J. P., Drake, J. W. Exogenous control of morphogenesis in isolated Fundulus blastoderms by 
nutrient chemical factors. The Journal of Experimental Zoology. 132 (2), 311-347 (1956).

16. Grinblat, Y., Lane, M. E., Sagerström, C., Sive, H. Analysis of zebrafish development using explant culture assays. Methods in Cell Biology. 59, 127-156 (1999).

17. Sagerström, C. G., Grinblat, Y., Sive, H. Anteroposterior patterning in the zebrafish, Danio rerio: an explant assay reveals inductive and suppressive cell interactions. Development. 122 (6), 1873-1883 (1996).

18. Sagerström, C. G., Gammill, L. S., Veale, R., Sive, H. Specification of the enveloping layer and lack of autoneuralization in zebrafish embryonic explants. Devolopmental Dynamics. 232 (1), 85-97 (2005).

19. Xu, P. F., Houssin, N., Ferri-Lagneau, K. F., Thisse, B., Thisse, C. Construction of a vertebrate embryo from two opposing morphogen gradients. Science. 344 (6179), 87-89 (2014).

20. Williams, M. L. K., Solnica-Krezel, L. Nodal and planar cell polarity signaling cooperate to regulate zebrafish convergence and extension gastrulation movements. eLife. 9 (2020).

21. de Olivera-Melo, M., Xu, P. F., Houssin, N., Thisse, B., Thisse, C. Generation of ectopic morphogen gradients in the Zebrafish blastula. Methods in Molecular Biology. 1863, 125-141 (2018).

22. Sepich, D. S., Calmelet, C., Kiskowski, M., SolnicaKrezel, L. Initiation of convergence and extension movements of lateral mesoderm during zebrafish gastrulation. Devolopmental Dynamics. 234 (2), 279-292 (2005).
23. Gritsman, K. et al. The EGF-CFC protein one-eyed pinhead is essential for nodal signaling. Cell. 97 (1), 121-132 (1999).

24. Feldman, B. et al. Zebrafish organizer development and germ-layer formation require nodal-related signals. Nature. 395 (6698), 181-185 (1998).

25. Spemann, H., Manngold, H. Uber inducktion von embryoalanlangen durch implantation artfremder organisatoren. Archiv für mikroskopische Anatomie und Entwicklungsmechanik. 100, 599-638 (1924).

26. Mangold, O. Über die Induktionsfähigkeit der verschiedenen Bezirke der Neurula von Urodelen. Naturwissenschaften. 21 (43), 761-766 (1933).

27. Shih, J., Fraser, S. E. Characterizing the zebrafish organizer: microsurgical analysis at the early-shield stage. Development. 122 (4), 1313-1322 (1996).

28. Agathon, A., Thisse, C., Thisse, B. The molecular nature of the zebrafish tail organizer. Nature. 424 (6947), 448-452 (2003).

29. Tacke, L., Grunz, H. Close juxtaposition between inducing chordamesoderm and reacting neuroectoderm is a prerequisite for neural induction in Xenopus laevis. Cell Death and Differentiation. 24 (1), 33-43 (1988).

30. Swanhart, L. M. et al. Characterization of an Ihx1a transgenic reporter in zebrafish. The International Journal of Developmental Biology. 54 (4), 731-736 (2010).

31. Williams, M. L. K., Solnica-Krezel, L. A mesodermindependent role for Nodal signaling in convergence \& extension gastrulation movements.BioRxiv. (2019).

32. Londin, E. R., Niemiec, J., Sirotkin, H. I. Chordin, FGF signaling, and mesodermal factors cooperate in 
zebrafish neural induction. Developmental Biology. 279

(1), 1-19 (2005).

33. Warmflash, A., Sorre, B., Etoc, F., Siggia, E. D., Brivanlou, A. H. A method to recapitulate early embryonic spatial patterning in human embryonic stem cells. Nature Methods. 11 (8), 847-854 (2014).

34. Veenvliet, J. V. et al. Mouse embryonic stem cells selforganize into trunk-like structures with neural tube and somites. Science. 370 (6522) (2020).

35. Beccari, L. et al. Multi-axial self-organization properties of mouse embryonic stem cells into gastruloids. Nature. 562 (7726), 272-276 (2018).

36. Moris, N. et al. An in vitro model of early anteroposterior organization during human development. Nature. $\mathbf{5 8 2}$ (7812), 410-415 (2020).

37. van den Brink, S. C. et al. Single-cell and spatial transcriptomics reveal somitogenesis in gastruloids. Nature. 582 (7812), 405-409 (2020). 